\title{
Aspectos Generales del Perfil Proteómico del Echinococcus granulosus
}

\author{
General Aspects of the Proteomic Profile of Echinococcus granulosus
}

\author{
Carlos Manterola ${ }^{1,2}$; Nayely García ${ }^{1} \&$ Claudio Rojas ${ }^{1}$
}

MANTEROLA, C.; GARCÍA, N. \& ROJAS, R. Aspectos generales del perfil proteómico del Echinococcus granulosus. Int. J. Morphol., 37(2):773-779, 2019.

RESUMEN: La información disponible referente a las características proteómicas del E. granulosus es escasa (no supera los 50 estudios publicados); y nos parece que la identificación proteómica, podría mejorar la comprensión de algunas características bioquímicas e inmunológicas de la Equinococosis Quística (EQ). De tal modo que el proteoma de E. granulosus aún no está bien descrito. Sólo existen reportes de algunas secuencias de proteínas. El objetivo de este manuscrito fue comentar algunos aspectos de la evidencia existente respecto de los estudios del perfil proteómico del E. granulosus. Se recomienda el estudio de al menos el quiste y su pared, el líquido hidatídico y la víscera del hospedero. Para ello, existen metodologías que han sido empleadas para estudiar las características proteómicas de la EQ. Entre ellas, destacan SDS-PAGE, electroforesis bidimensional combinada con Western Blot, inmunoanálisis, y espectrometría de masas mediante técnicas MALDI-TOF. Se han identificado una serie de proteínas en muestras de EQ. Algunas de ellas, asociadas a procesos inmunológicos, de gluconeogénesis, glucogenolisis y glucogénesis. Por otra parte, se ha documentado la liberación de exosomas al líquido hidatídico por parte de los protoescólex y la capa germinativa; estructuras en las que se han identificado factores de virulencia asociados con la supervivencia del quiste. No obstante lo anteriormente señalado, se requiere de múltiples estudios exhaustivos en la materia para comprender mejor la caracterización perfil proteómico del E. granulosus.

PALABRAS CLAVE: Echinococcus granulosus; Proteómica.

\section{INTRODUCCIÓN}

La hidatidosis o equinococosis quística (EQ), es una zoonosis que representa un problema de salud pública con importantes repercusiones socioeconómicas. Es causada por la infección con el estadio larvario de Echinococcus granulosus (E. granulosus); parte del phylum Platyhelminthes, clase Cestoda; subclase Eucestoda, orden Cyclophyllidea, familia Taeniidae, género Echinococcus (Romig et al., 2015).

Como todos los euctos, estos organismos carecen de tiene intestino, por lo que, el intercambio de nutrientes y desechos se produce a través del tegumento o cubierta de la célula externa.

El hombre, se infecta por la ingestión accidental de huevos de E. granulosus provenientes de heces de cánidos infectados. Sin embargo, se ha reportado que Echinococcus spp, pueden infectar a las personas a través de alimentos o por contacto directo con animales.
Existe cierta evidencia que sugiere que algunos genotipos serían mas virulentos para el hombre humanos que otros (Cucher et al., 2016).

Este interesante fenómeno, propio de la biología del E. granulosus; se ha estudiado mediante técnicas de biología molecular, basadas en la reacción en cadena de la polimerasa (PCR) y el análisis secuencial del ADN; lo que ha permitido caracterizar la complejidad de estos de céstodos denominados E. granulosus sensu lato (sl), compuesto por: E. granulosus ss (genotipos G1-G3), E. equinus (G4), E. ortleppi (G5) y E. canadensis (G6-G10); con variaciones fenotípicas, que se pueden evidenciar en las características del ciclo biológico, especificidad del huésped intermedio, patrón de desarrollo, patogenicidad, antigenicidad, dinámica de transmisión y; por consiguiente, en las medidas necesarias para controlar la enfermedad (Bowles et al., 1992; Bowles \& McManus, 1993a,b; Daniel Mwambete et al., 2004; Ahn et al., 2015).

\footnotetext{
${ }^{1}$ Programa de Doctorado en Ciencias Médicas, Universidad de La Frontera, Chile.

${ }^{2}$ Centro de Excelencia en Estudios Morfológicos y Quirúrgicos (CEMyQ), Universidad de La Frontera, Chile.
} 
La información disponible referente a las características proteómicas del E. granulosus es escasa (no supera los 50 estudios publicados); y nos parece que la identificación proteómica, podría mejorar la comprensión de algunas características bioquímicas e inmunológicas de la EQ (M'rad et al., 2005; Cucher et al.; Manterola \& Otzen, 2016; Avila et al., 2017). De tal modo que el proteoma de EQ aún no está bien descrito. Sólo existen reportes de algunas secuencias de proteínas (UniProt, 2019; NCBI, 2019).

El objetivo de este manuscrito fue comentar aspectos de la evidencia existente respecto de los estudios del perfil proteómico del EQ.

\section{MATERIAL Y MÉTODO}

\section{Diseño: Revisión narrativa.}

Población: Se incluyeron todo tipo de estudios publicados hasta febrero de 2019, sin restricción idiomática, que incluyeran población humana o animal, que aportasen en los métodos de cuantificación proteómica de EQ. No se consideraron criterios de exclusión.

Fuentes de información: Se revisaron los siguientes metabuscadores, bibliotecas y bases de datos: Trip Database, BIREME-BVS, SciELO, WoS, MEDLINE, EMBASE y SCOPUS.

Estrategias de búsqueda: En las fuentes antes señaladas, se realizaron búsquedas sensibles utilizando los términos "Echinococcus granulosus"[Mesh]; "Echinococcus granulosus/genetics"[Mesh]; "Echinococcus granulosus/ metabolism"[Mesh]; "Proteomics"[Mesh]; "Proteomics/ methods"[Mesh]; y conectores booleanos (AND y OR); utilizando estrategias adaptadas a cada fuente de información.

Protocolo de estudio: La recuperación de documentos se cerró el 28 de febrero de 2019. De los artículos seleccionados, se analizaron en orden progresivo títulos, resúmenes y artículo en extenso, en búsqueda del cumplimiento de los criterios de inclusión y exclusión.

Variables de interés: Las variables de interés fueron material de estudio necesario para determinar proteómica, métodos más frecuentemente utilizados, y proteínas identificadas.

Herramientas estadísticas: No se utilizan.

Aspectos éticos: Se realizó enmascaramiento de autores y centros.

\section{RESULTADOS}

Con las estrategias utilizadas en las fuentes de información, se lograron recuperar 35 artículos, los cuales fueron analizados a título y resumen; lo que permitió seleccionar un total de 15 artículos, los que fueron revisados a texto completo, los que constituyen el insumo del presente reporte. Con ellos, se sintetiza la evidencia respecto de las siguientes definiciones:

Material de estudio: Se recomienda el estudio de al menos el quiste y su pared, el líquido hidatídico y la víscera del hospedero; a partir de los cuales se puede realizar análisis de datos y listar las proteínas especificas encontradas (Ahn et al.) (Fig. 1).

En muestras de EQ, se pueden estudiar las características proteómicas del hospedero, de la pared del quiste, del líquido hidatídico y de los protoescólex. Es así como, existe evidencia de cambios en la expresión de proteínas de los protoescólex, en respuesta a factores externos, como el tratamiento antihelmíntico (Chemale et al., 2003). Algo similar a lo descrito para la composición de proteínas del líquido hidatídico en diferentes ubicaciones anatómicas del hospedador (Aziz et al., 2011; Cui et al., 2013).

Métodos utilizados: Uno de los métodos más utilizados para el análisis de proteínas es el SDS-PAGE (electroforesis en gel de poliacrilamida con dodecilsulfato sódico) (Aziz et $a l$.). De hecho, ya ha sido utilizado para el estudio de líquido quiste hidatídico (Fig. 2).

La electroforesis bidimensional combinada con Western Blot, así como el inmunoanálisis, también han jugado un rol clave para el análisis proteómico incluso en la búsqueda de antígenos de E. granulosus específicos (Li \& Zhao, 2012).

Sin embargo, el bioanálisis de las técnicas de espectrometría de masas mediante técnicas MALDI-TOF MS (Matrix-assited laser desorption/ionization time-of-flight mass spectrometry) se convirtió en un recurso de referencia para la proteómica y la identificación de microorganismos en parasitología clínica; entre otras, en EQ. De hecho, con análisis MALDI-TOF/TOF, se ha demostrado la expresión cambiante de proteínas de EQ, generando alteraciones en los niveles de expresión de éstas en el suero de pacientes con EQ (Sadjjadi et al., 2018).

Proteínas identificadas: Se han identificado alrededor de 80 proteínas diferentes en muestras de EQ (Fig. 3). Algunas de ellas, asociadas a los procesos de gluconeogénesis, 


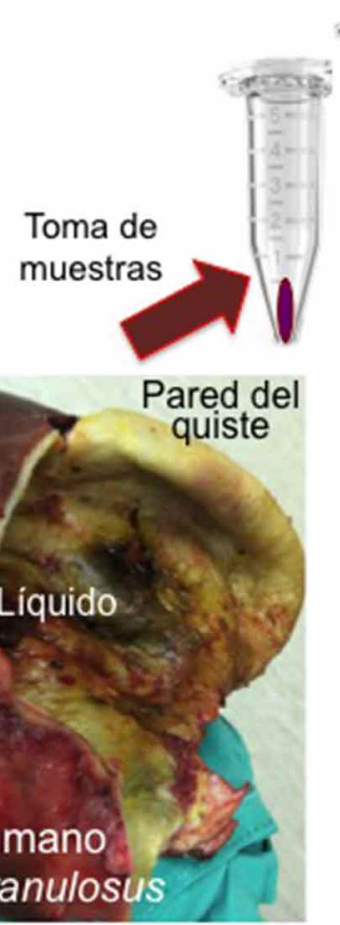

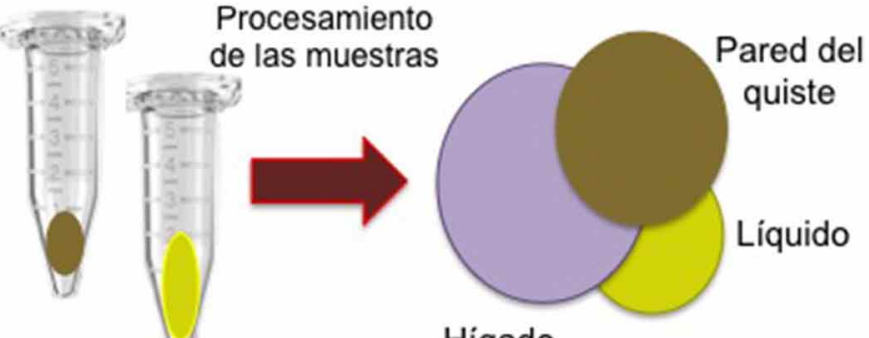

Hígado

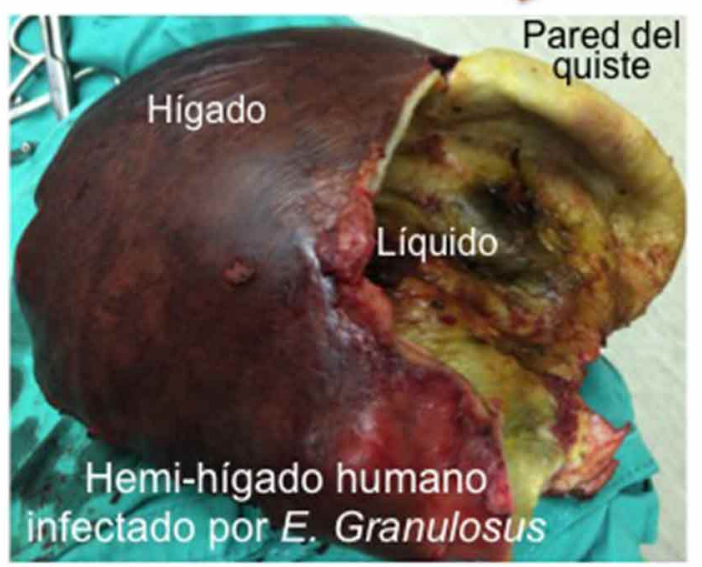

Fig. 1. Análisis de un hígado humano infectado por E. granulosus. Las regiones de interés son el quiste y su pared, el líquido hidatídico y el hígado. Una vez obtenida las muestras, se realiza el procesamiento bioquímico, los péptidos proteolíticos digeridos se someten a cromatografía líquida de masas, espectrometría de masas y análisis de datos, para posteriormente adjuntarlos al listado de proteínas específicas.

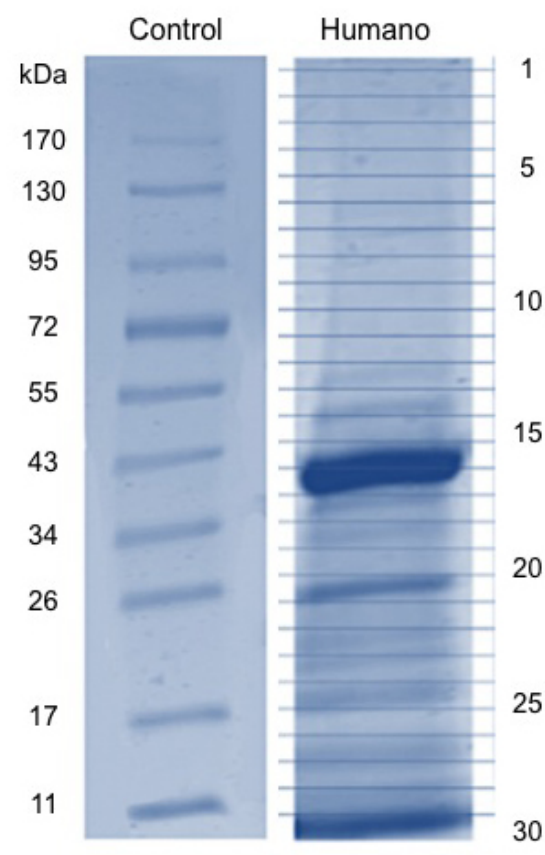

Fig. 2. SDS-PAGE de líquido de quiste hidatídico humano, después de tinción con azul de Coomassie. Se evidencian proteínas que se tiñen de $170 \mathrm{kDa}$ a $11 \mathrm{kDa}$. El carril de la izquierda corresponde al control y el de la derecha a una muestra de hidatidosis humana. El sistema de numeración utilizado para las discos de gel se aprecian a la derecha de la muestra.

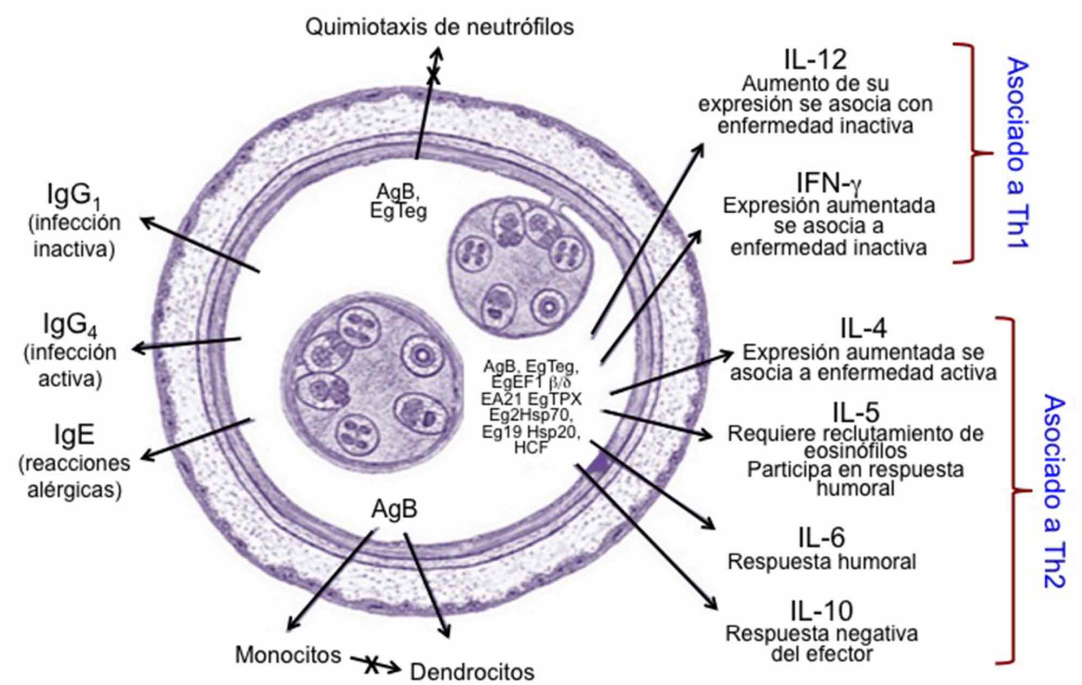

Fig. 3. Principales componentes de la respuesta inmunológica al líquido hidatídico en el huésped. Moduladores inmunológicos derivados del $E$. granulosus y principales citoquinas que regulan esta respuesta. Moléculas derivadas del parásito (AgB, EgTeg y EgEF-1b/d), pueden provocar activación predominantemente Th2; mientras que EgTPx y otros, pueden provocar activación de células Th1/Th2 de forma concomitante. 
glucogenolisis y glucogénesis (Aziz et al.; Cui et $a l$.). En una experiencia china (Aziz et al.), el análisis de material de quistes hidatídicos fértiles provenientes de tres pacientes intervenidos por hidatidosis en Xinjiang; permitió reconocer algunas proteínas presentes en el líquido hidatídico, conociendo de este modo la composición de las proteínas del parásito (Tabla I); así como algunas proteínas presentes en los hospederos que han sido estudiados (Tabla II).

Por otra parte, se ha observado que al comparar el perfil proteómico del líquido hidatídico, existen diferencias en la expresión de la proteína y adaptación del metacestodo en su entorno huésped, según la localización de la lesión quística; así como también isoformas en las fracciones citosólica, nuclear, mitocondrial y microsomal (Alvite \& Esteves, 2016; Zeghir-Bouteldja et al., 2017). Por ende, se puede señalar que existe evidencia orientativa de asociación de proteínas específicas a diversos tejidos, fenotipos celulares, estadios del parásito y procesos patobioquímicos, así como procesos inmunológicos en la EQ (Longuespée et al., 2017).
Tabla I. Algunas proteínas parasitarias identificadas mediante espectrometría de masas en tándem, en líquido de quistes hidatídicos humanos por infección con E. granulosus.

\begin{tabular}{lll}
\hline ID & Descripción & $\begin{array}{l}\text { Recuento espectral } \\
(\% \text { de cobertura })\end{array}$ \\
\hline $\begin{array}{l}\text { Enzimas metabólicas } \\
21731\end{array}$ & $\begin{array}{l}\text { Fructosa-bifos fato aldolasa } \\
38622\end{array}$ & $5(18,0)$ \\
37276 & $\begin{array}{l}\text { Fosfoenolpiruvato } \\
\text { Glutation S-transferasa }\end{array}$ & $4(8,0)$ \\
82276 & Malato deshidrogenasa & $2(6,0)$ \\
38910 & Glucosa fosfatasa & $5(10,0)$ \\
Cito esqueleto & & \\
14260 & Actina & $6(10,0)$ \\
82514 & Proteína tegumentaria & $2(21,0)$ \\
Proteólisis & & \\
40093 & Antígeno 5 de 38 kDa & $19(25,0)$ \\
Misceláneas & & \\
11212 & Histona H4 & $3(29,0)$ \\
14646 & Poliubiquitina & $3(8,0)$ \\
16815 & Proteína amiloidea beta A4 & $2(13,0)$ \\
83259 & Antígeno B & $5(65,0)$ \\
36286 & Antígeno B subunidad 4 & $4(36,0)$ \\
\hline
\end{tabular}

Tabla II. Proteínas del huésped identificadas mediante espectrometría de masas en tándem, en líquido de quistes hidatídicos humanos por infección con E. granulosus.

\begin{tabular}{|c|c|c|}
\hline ID & Descripción & $\begin{array}{c}\text { Recuento espectral } \\
\text { (\% de cobertura) }\end{array}$ \\
\hline \multicolumn{3}{|c|}{ Enzimas metabólicas } \\
\hline 1 & Carbónico anhidrasa 2 & $3(14,0)$ \\
\hline 2 & Carbónico anhidrasa I & $6(33,0)$ \\
\hline 5 & Piruvato quinasa & $4(14,0)$ \\
\hline 6 & Enolasa & $3(8,0)$ \\
\hline 7 & Biliverdina reductasa $\mathrm{B}$ & $2(14,0)$ \\
\hline 8 & Triosefosfato isomerasa 1 & $2(22,0)$ \\
\hline \multicolumn{3}{|c|}{ Respuesta inmunológica } \\
\hline 16 & $\operatorname{IgA} 1$ & $2(10,0)$ \\
\hline 17 & $\operatorname{IgG}$ & $10(16,0)$ \\
\hline 18 & $\mathrm{IgG}$ & $5(7,0)$ \\
\hline 22 & $\operatorname{IgM}$ & $3(17,0)$ \\
\hline 23 & Ig kappa & $7(44,0)$ \\
\hline 28 & Alfa-1-ácido glicoproteína & $5(22,0)$ \\
\hline 29 & Zinc-alfa2-glicoproteína & $2(7,0)$ \\
\hline 30 & Complemento $\mathrm{C} 3$ & $6(4,0)$ \\
\hline \multicolumn{3}{|c|}{ Misceláneas } \\
\hline 68 & Galectina-7 (unión de azúcar) & $5(40,0)$ \\
\hline 69 & Ciclofilina (actividad de peptidil-prolil cis-trans isomerasa) & $3(18,0)$ \\
\hline 70 & Histona $1 \mathrm{H} 2 \mathrm{AD}$ & $2(37,0)$ \\
\hline 71 & Haptoglobina (proteólisis) & $3(12,0)$ \\
\hline 72 & Factor 1 eucariótico de translación elongación & $2(5,0)$ \\
\hline 73 & Proteína ribosomal S5 (estructural) & $2(14,0)$ \\
\hline
\end{tabular}


Por otra parte, se ha verificado que los componentes de un quiste hidatídico, en especial los protoescólex y la capa germinativa; producen y liberan exosomas en el líquido hidatídico; estructuras en las que se han identificado factores de virulencia asociados con la supervivencia del quiste, incluidos antígenos y peptidasas altamente inmunogénicos (Siles-Lucas et al., 2018). En este sentido, se han desarrollado investigaciones de productos excretores y secretores de las proteínas antigénicas del E. granulosus en su forma adulta en cánidos (Wang et al., 2015).

\section{DISCUSIÓN}

El enfoque inmunoproteómico para el diagnóstico de enfermedades infecciosas humanas es una herramienta muy interesante. Los primeros pasos en este sentido se dieron a fines de la década de los 80's y principios de los 90's se inició el estudio y caracterización de proteínas en membranas y muestras de líquido hidatídico, utilizando como fuen- te proteínas de metacéstodo para inmunodiagnóstico (Gasser et al., 1989, 1991).

Las herramientas proteómicas y el análisis bioinformático de sueros de pacientes con niveles de expresión de proteínas relacionados en este caso con hidatidosis; podrían ayudar a realizar diagnóstico más válidos y confiables; lo que redundaría en un incremento en la calidad de los procesos de seguimiento posteriores a un tratamiento (Celik et al., 2009; Sadjjadi et al.).

El diagnóstico de moléculas liberadas en el suero de pacientes con hidatidosis, podría utilizarse como objetivo diagnóstico-terapéutico; por ende, el análisis del suero de sujetos con equinococosis quística y su comparación con individuos normales, podría ayudar a una mejor comprensión de la relación huésped-parásito (Virginio et al., 2012).

Siguiendo esta línea de trabajo, es que no parece lejano el poder desarrollar una nueva vacuna para el huésped intermediario a partir del perfil proteómico de los genotipos más frecuentes que afectan un territorio (en el caso nuestro G1,
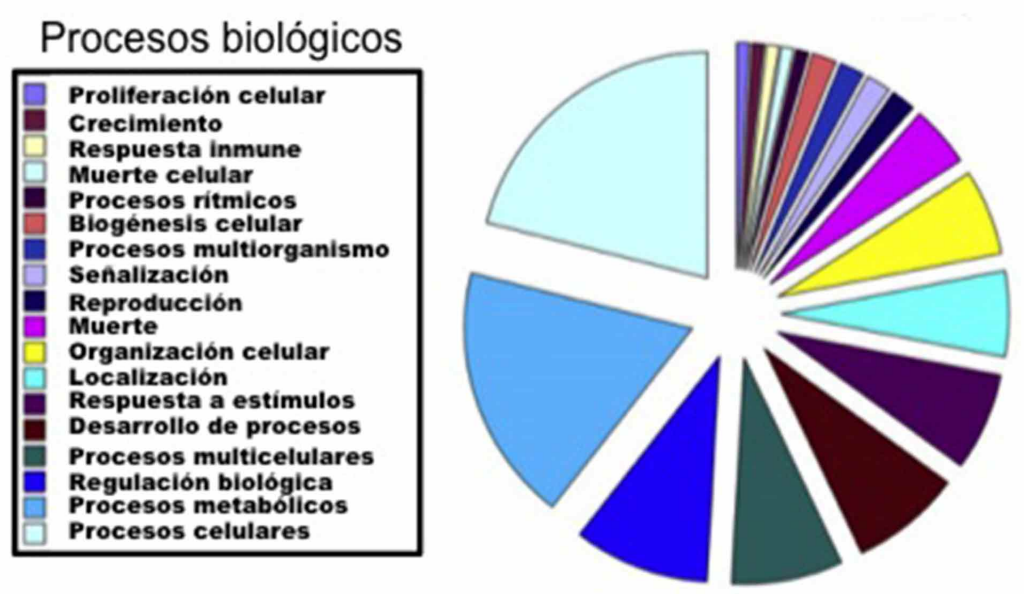

\section{Función molecular}

\begin{tabular}{|l|} 
Actividad enzimática \\
Actividad transcripción \\
Uniones \\
Actividad catalítica \\
Actividad antioxidante \\
Actividad transportadora \\
Actividad estructural \\
\hline
\end{tabular}

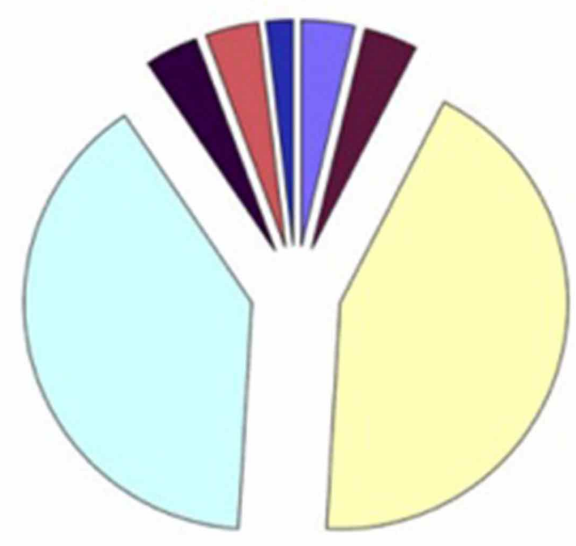

Fig. 4. Términos ontológicos del proceso biológico, función molecular y subcategorías de componentes celulares de genes asociados con proteínas del parásitos identificadas en el fluido de un quiste hidatídico mediante Blast2Go.
G3, G5 y G6) (Manterola et al., 2008; Espinoza et al., 2014; Alvarez Rojas et al., 2017; Corrêa et al., 2018; Hernández et al., 2019); puesto que la existente (Providean Hidatil EG 95Ò), fue desarrollada por utilizando una pequeña fracción proteica de la membrana exterior del parásito; por lo cual presenta una serie de limitaciones, por ejemplo no otorgar similar cobertura en otros genotipos como G6 y G7, pues fue desarrollada para G1; y no da cobertura a la gran variabilidad de haplotipos descritos (Chow et al., 2008; Alvarez Rojas et al., 2013).

Por otra parte, se han asignado términos de ontología génica a las proteínas identificadas (Fig. 4); sobre la base de la similitud utilizando Blast2Go (software de bioinformática para la anotación funcional automática de nuevos datos de secuencias) (Götz et al., 2008).

No obstante lo anteriormente señalado, se requiere de múltiples estudios exhaustivos en la materia para comprender mejor la caracterización perfil proteómico del EG; lo que indudablemente contribuirá en una serie de aspectos del conocimiento de la enfermedad (morfología y fisiología del parásito, virulencia, diagnóstico precoz, prevención y tratamiento). 
MANTEROLA, C.; GARCÍA, N. \& ROJAS, R. General aspects of the proteomic profile of Echinococcus granulosus. Int. J. Morphol., 37(2):773-779, 2019

SUMMARY: The information available regarding the proteomic characteristics of E. granulosus is scarce; and it seems that the proteomic identification could improve the understanding of some biochemical and immunological characteristics of cystic echinococcosis (CE). So, the proteome of E. granulosus is still not well described yet. There are only reports of some protein sequences. The objective of this manuscript was to comment on some aspects of the existing evidence regarding studies of the proteomic profile of E. granulosus. The study of at least the cyst and its wall, the hydatid fluid and the viscera of the host are recommended. There are a series of methodologies that have been used to study the proteomic characteristics of EQ. These include SDS-PAGE, bidimensional electrophoresis combined with Western Blot, immunoassay, and mass spectrometry using MALDI-TOF techniques. A series of proteins have been identified in CE samples. Some of them, associated with immune response, gluconeogenesis, glycogenolysis and glycogenesis. On the other hand, release of exosomes to the hydatid fluid by protoescolex and germinative layer has been documented (associated virulence factors have been identified in these structures). Notwithstanding the foregoing, it requires multiple exhaustive studies in the field to better understand the characterization of the proteomic profile of E. granulosus.

KEY WORD: "Echinococcus granulosus"[Mesh]; "Echinococcus granulosus/genetics"[Mesh]; "Echinococcus granulosus/metabolism"[Mesh]; "Proteomics"[Mesh]; "Proteomics/methods"[Mesh].

\section{REFERENCIAS BIBLIOGRÁFICAS}

Ahn, C. S.; Han, X.; Bae, Y. A.; Ma, X.; Kim, J. T.; Cai, H.; Yang, H. J.; Kang, I.; Wang, H. \& Kong, Y. Alteration of immunoproteome profile of Echinococcus granulosus hydatid fluid with progression of cystic echinococcosis. Parasit. Vectors, 8:10, 2015.

Alvarez Rojas, C. A.; Ebi, D.; Paredes, R.; Acosta-Jamett, G.; Urriola, N.; Roa, J. C.; Manterola, C.; Cortes, S.; Romig, T.; Scheerlinck, J. P. \& Lightowlers, M. W. High intraspecific variability of Echinococcus granulosus sensu stricto in Chile. Parasitol. Int., 66(2):112-5, 2017.

Alvarez Rojas, C. A.; Gauci, C. G. \& Lightowlers, M. W. Antigenic differences between the EG95-related proteins from Echinococcus granulosus $\mathrm{G} 1$ and $\mathrm{G} 6$ genotypes: implications for vaccination. Parasite Immunol., 35(2):99-102, 2013.

Alvite, G. \& Esteves, A. Echinococcus granulosus fatty acid binding proteins subcellular localization. Exp. Parasitol., 164:1-4, 2016.

Avila, H. G.; Santos, G. B.; Cucher, M. A.; Macchiaroli, N.; Pérez, M. G.; Baldi, G.; Jensen, O.; Pérez, V.; López, R.; Negro, P.; et al. Implementation of new tools in molecular epidemiology studies of Echinococcus granulosus sensu lato in South America. Parasitol. Int., 66(3):250-7, 2017.

Aziz, A.; Zhang, W.; Li, J.; Loukas, A.; McManus, D. P. \& Mulvenna, J. Proteomic characterisation of Echinococcus granulosus hydatid cyst fluid from sheep, cattle and humans. J. Proteomics, 74(9):1560-72, 2011.

Bowles, J. \& McManus, D. P. Molecular variation in Echinococcus. Acta Trop., 53(3-4):291-305, 1993b.
Bowles, J. \& McManus, D. P. NADH dehydrogenase 1 gene sequences compared for species and strains of the genus Echinococcus. Int. J. Parasitol., 23(7):969-72, 1993a.

Bowles, J.; Blair, D. \& McManus, D. P. Genetic variants within the genus Echinococcus identified by mitochondrial DNA sequencing. Mol. Biochem. Parasitol., 54(2):165-73, 1992.

Celik, T.; Akarsu, G. A.; Güngör, C.; Colak, C. \& Ormeci, N. Evaluation of antibodies against hydatid cyst fluid antigens in the post-treatment followup of cystic echinococcosis patients. Med. Sci. Monit., 15(4):CR170-6, 2009.

Chemale, G.; van Rossum, A. J.; Jefferies, J. R.; Barrett, J.; Brophy, P. M.; Ferreira, H. B. \& Zaha, A. Proteomic analysis of the larval stage of the parasite Echinococcus granulosus: causative agent of cystic hydatid disease. Proteomics, 3(8): 1633-6, 2003.

Chow, C.; Gauci, C. G.; Vural, G.; Jenkins, D. J.; Heath, D. D.; Rosenzvit, M. C.; Harandi, M. F. \& Lightowlers, M. W. Echinococcus granulosus: variability of the host-protective EG95 vaccine antigen in G6 and G7 genotypic variants. Exp. Parasitol., 119(4):499-505, 2008.

Corrêa, F.; Stoore, C.; Horlacher, P.; Jiménez, M.; Hidalgo, C.; Alvarez Rojas, C. A.; Figueiredo Barros, G.; Bunselmeyer Ferreira, H.; Hernández, M.; Cabrera, G. ; et al. First description of Echinococcus ortleppi and cystic echinococcosis infection status in Chile. PLoS One, 13(5):e0197620, 2018.

Cucher, M. A.; Macchiaroli, N.; Baldi, G.; Camicia, F.; Prada, L.; Maldonado, L.; Avila, H. G.; Fox, A.; Gutiérrez, A.; Negro, P.; et al. Cystic echinococcosis in South America: systematic review of species and genotypes of Echinococcus granulosus sensu lato in humans and natural domestic hosts. Trop. Med. Int. Health, 21(2):166-75, 2016.

Cui, S. J.; Xu, L. L.; Zhang, T.; Xu, M.; Yao, J.; Fang, C. Y.; Feng, Z.; Yang, P. Y.; Hu, W. \& Liu, F. Proteomic characterization of larval and adult developmental stages in Echinococcus granulosus reveals novel insight into host-parasite interactions. J. Proteomics, 84:158-75, 2013.

Daniel Mwambete, K.; Ponce-Gordo, F. \& Cuesta-Bandera, C. Genetic identification and host range of the Spanish strains of Echinococcus granulosus. Acta Trop., 91(2):87-93, 2004

Espinoza, S.; Salas, A. M.; Vargas, A.; Freire, V.; Diaz, E.; Sánchez, G. \& Venegas, J. Detection of the G3 genotype of Echinococcus granulosus from hydatid cysts of Chilean cattle using COX1 and ND1 mitochondrial markers. Parasitol. Res., 113(1):139-47, 2014.

Gasser, R. B.; Lightowlers, M. W. \& Rickard, M. D. Echinococcus granulosus: antigenic proteins in oncospheres and on the surface of protoscoleces identified by serum antibodies from infected dogs. Res. Vet. Sci., 50(3):340-5, 1991

Gasser, R. B.; Lightowlers, M. W. \& Rickard, M. D. Identification of protein components of Echinococcus granulosus protoscolex antigens for specific serodiagnosis of E. granulosus infection in dogs. Parasite Immunol., 11(3):279-91, 1989.

Götz, S.; García-Gómez, J. M.; Terol, J.; Williams, T. D.; Nagaraj, S. H.; Nueda, M. J.; Robles, M.; Talón, M.; Dopazo, J. \& Conesa, A. Highthroughput functional annotation and data mining with the Blast2GO suite. Nucleic Acids Res., 36(10):3420-35, 2008.

Hernández F.; Verdugo, C.; Cárdenas, F.; Sandoval, R.; Morales, N.; Olmedo, P.; Bahamonde, A.; Aldridge, D. \& Acosta-Jamett, G. Echinococcus granulosus in the Endangered Patagonian Huemul (Hippocamelus bisulcus). J. Wildl. Dis., 2019. En prensa.

Li, Z. J. \& Zhao, W. Analysis of protoscoleces-specific antigens from Echinococcus granulosus with proteomics combined with Western blot. Biomed. Environ. Sci., 25(6):718-23, 2012.

Longuespée, R.; Casadonte, R.; Kriegsmann, M.; Wandernoth, P.; Lisenko, K.; Mazzucchelli, G.; Becker, M. \& Kriegsmann, J. Proteomic investigation of human cystic echinococcosis in the liver. Mol. Biochem. Parasitol., 211:9-14, 2017.

Mrad, S.; Filisetti, D.; Oudni, M.; Mekki, M.; Belguith, M.; Nouri, A.; Sayadi, T.; Lahmar, S.; Candolfi, E.; Azaiez R.; et al. Molecular evidence of ovine (G1) and camel (G6) strains of Echinococcus granulosus in Tunisia and putative role of cattle in human contamination. Vet. Parasitol., 129(34):267-72, 2005. 
Manterola, C. \& Otzen, T. Molecular epidemiology of cystic echinococcosis: genotypic characterization in humans and different livestock. Int. J. Morphol., 34(4):1472-81, 2016.

Manterola, C.; Benavente, F.; Melo, A.; Vial, M. \& Roa, J. C. Description of Echinococcus granulosus genotypes in human hydatidosis in a region of southern Chile. Parasitol. Int., 57(3):342-6, 2008.

NCBI. Protein. Bethesda, National Center for Biotechnology Information, U. S. National Library of Medicine, 2019. Disponible en: https:// www.ncbi.nlm.nih.gov/protein

Romig, T.; Ebi, D. \& Wassermann, M. Taxonomy and molecular epidemiology of Echinococcus granulosus sensu lato. Vet. Parasitol., 213(3-4):76-84, 2015.

Sadjjadi, F. S.; Rezaie-Tavirani, M.; Ahmadi, N. A.; Sadjjadi, S. M. \& Zali, H. Proteome evaluation of human cystic echinococcosis sera using two dimensional gel electrophoresis. Gastroenterol. Hepatol. Bed Bench, 11(1):75-82, 2018.

Siles-Lucas, M.; Sánchez-Ovejero, C.; González-Sánchez, M.; González, E.; Falcón-Pérez, J. M.; Boufana, B.; Fratini, F.; Casulli, A. \& Manzano-Román, R. Isolation and characterization of exosomes derived from fertile sheep hydatid cysts. Vet. Parasitol., 236:22-33, 2018.

UniProt. UniProt Consortium, 2019. Disponible en: http://www.uniprot.org Virginio, V. G.; Monteiro, K. M.; Drumond, F.; de Carvalho, M. O.; Vargas, D. M.; Zaha, A. \& Ferreira, H. B. Excretory/secretory products from in vitro-cultured Echinococcus granulosus protoscoleces. Mol. Biochem. Parasitol., 183(1):15-22, 2012.

Wang, Y.; Xiao, D.; Shen, Y.; Han, X.; Zhao, F.; Li, X.; Wu, W.; Zhou, H.; Zhang, J. \& Cao, J. Proteomic analysis of the excretory/secretory products and antigenic proteins of Echinococcus granulosus adult worms from infected dogs. B. M. C. Vet. Res., 11:119, 2015.

Zeghir-Bouteldja, R.; Polomé, A.; Bousbata, S. \& Touil-Boukoffa, C. Comparative proteome profiling of hydatid fluid from Algerian patients reveals cyst location-related variation in Echinococcus granulosus. Acta Trop., 171:199-206, 2017.

\author{
Dirección para correspondencia: \\ Dr. Carlos Manterola \\ Departamento de Cirugía y CEMyQ \\ Universidad de La Frontera \\ Manuel Montt 112, oficina 408 \\ Temuco \\ CHILE
}

E-mail: carlos.manterola@ufrontera.cl

Recibido : 10-01-2019

Aceptado : 22-03-2019 